\title{
Evolução da ferramenta App Inventor para suportar o ensino de design interface na Educação Básica
}

\author{
Fernando da Cruz Pinheiro" ${ }^{1}$, Christiane Gresse von Wangenheim ${ }^{1}$, Jean Hauck ${ }^{1}$, \\ Miriam Nathalie F. Ferreira ${ }^{2}$, \\ ${ }^{1}$ Deparatamento de Informática e Estatística - Universidade Federal de Santa Catarina \\ ${ }^{2}$ Departmento de Expressão Gráfica - Universidade Federal de Santa Catarina \\ \{fernando.pinheiro, nathalie.fortuna\} eposgrad.ufsc.br, \\ c.wangenheim@ufsc.br, jean.hauck@ufsc.br
}

\begin{abstract}
App Inventor is widely used to teach programming through the development of mobile applications in K-12. It allows to design the user interface as well as to program the functionality of apps. However, shortcomings have been identified with respect to its support provided for designing user interfaces in alignment with a human-centered design process and visual design guides. This article presents enhancements of the App Inventor tool in order to facilitate teaching of user interface design in computing education. First results indicate that such improvements can have a positive impact on learning as well as empower students by becoming active creators of visually aesthetic and usable products.
\end{abstract}

Resumo. O App Inventor é usado para ensinar programação por meio do desenvolvimento de aplicativos móveis na Educação Básica. Ele permite projetar a interface do usuário, bem como programar sua funcionalidade. No entanto, foram identificadas deficiências em relação ao suporte para projetar interfaces em alinhamento com um processo de design centrado no usuário e guias de design visual. Este artigo apresenta aprimoramentos do App Inventor para facilitar o ensino do design da interface do usuário no ensino de computação. Primeiros resultados indicam que tais melhorias podem ter um impacto positivo na aprendizagem, bem como capacitar os alunos de criar produtos esteticamente atrativos e utilizáveis.

\section{Introdução}

Para ser um cidadão preparado num mundo permeado de tecnologia, é necessário formar criadores de novas soluções de tecnologias da informação (TI) (CSTA, 2017). Uma forma de popularizar a computação é iniciar o ensino de computação já na Educação Básica (CSTA, 2017). Isso tipicamente é realizado por meio do ensino de algoritmos e programação (Grover \& Pea, 2013), utilizando, p. ex. o desenvolvimento de aplicativos com App Inventor ${ }^{1}$. O App Inventor é uma ferramenta de código aberto, que permite a qualquer pessoa criar aplicativos para dispositivos Android por meio da programação baseada em blocos. Já existem várias unidades instrucionais para desenvolver aplicativos que focam basicamente somente no ensino de conceitos e práticas de programação (Pinheiro et al., 2018) (Grover \& Pea, 2013).

\footnotetext{
${ }^{1}$ App Inventor. http://appinventor.mit.edu.
} 
VIII Congresso Brasileiro de Informática na Educação (CBIE 2019)

Anais do XXV Workshop de Informática na Escola (WIE 2019)

Porém, uma qualidade importante de aplicativos é a usabilidade, que pode ser definida como a medida na qual um produto pode ser usado por usuários específicos, para alcançar objetivos específicos, com eficácia, eficiência e satisfação, em um contexto específico de uso (ISO/IEC 9241:210, 2011). Uma forma de melhorar a usabilidade consiste em abordar conceitos e práticas de design de interface de usuário (user interface - UI). As competências relacionadas ao design da UI são importantes não apenas para os profissionais de TI, mas para qualquer pessoa, pois estão relacionadas às habilidades do século XXI e incentivam os alunos a serem imaginativos, ensinando-os como aproveitar essa inventividade e colocá-la em prática (AIGA, 2013). Alguns países já começaram a incorporar o ensino de design na Educação Básica, como China e Coréia do Sul (West-Knights, 2017; Ahn, 2012) e primeiras unidades instrucionais com esse foco estão sendo propostas no Brasil (Pinheiro, 2019; Ferreira et al., 2019).

Atualmente, o App Inventor oferece um suporte básico para o design de interface incluindo diversos tipos de elementos de design de UI como botões, labels, etc., que podem ser configurados em relação a cor, tamanho, tipografia etc. Porém, observa se a falta de suporte de forma mais completa tanto para a documentação de artefatos como histórias de usuário e personas, quanto a um suporte ao design visual mais alinhado aos meta-princípios (Garrett, 2011; Schlatter \& Levinson, 2013) e guias de design visual como o Material Design (Google, 2019) e Web Content Accessibility Guidelines 2.0 (WCAG ${ }^{2}$. . Nesse contexto, este artigo apresenta melhorias no App Inventor, a fim de facilitar o ensino de conceitos e práticas de design de interface como parte do ensino de computação na Educação Básica.

\section{Metodologia de pesquisa}

O aprimoramento da ferramenta App Inventor foi realizado utilizando uma metodologia multi-método. Analisando unidades instrucionais que ensinam o design de UI com App Inventor e experiências práticas (Pinheiro, 2019; Ferreira et al., 2019), foram identificadas as oportunidades de melhorias. Essas foram desenvolvidas no App Inventor seguindo um processo de desenvolvimento iterativo e incremental (Basili \& Larman, 2003). Com base nas necessidades identificadas foram especificados os requisitos funcionais, que foram revisados por um grupo de especialistas da área de computação, design e educação com experiência prática. As funcionalidades foram modeladas e implementadas utilizando Java e oframework Google Web Toolkit $\left(\mathrm{GWT}^{3}\right)$. Foram realizados testes de unidade e de sistema, como também testes de usabilidade. As melhorias realizadas no App Inventor foram utilizadas em uma aplicação da unidade instrucional "Faça um app" (Pinheiro, 2019) por meio de um estudo de caso. O estudo de caso foi sistematicamente definido, planejado, executado e analisado seguindo Yin (2009). Dados foram coletados principalmente com base na avaliação de desempenho dos artefatos criados pelos alunos.

\section{Evolução do App Inventor}

Com o objetivo de suportar o ensino de design de UI na Educação Básica com App Inventor foram identificadas oportunidades de melhoria no desenvolvimento de apps (Figura 1).

\footnotetext{
2 https://www.w3.org/TR/2008/REC-WCAG20-20081211

${ }^{3}$ www.gwtproject.org
} 
VIII Congresso Brasileiro de Informática na Educação (CBIE 2019)

Anais do XXV Workshop de Informática na Escola (WIE 2019)

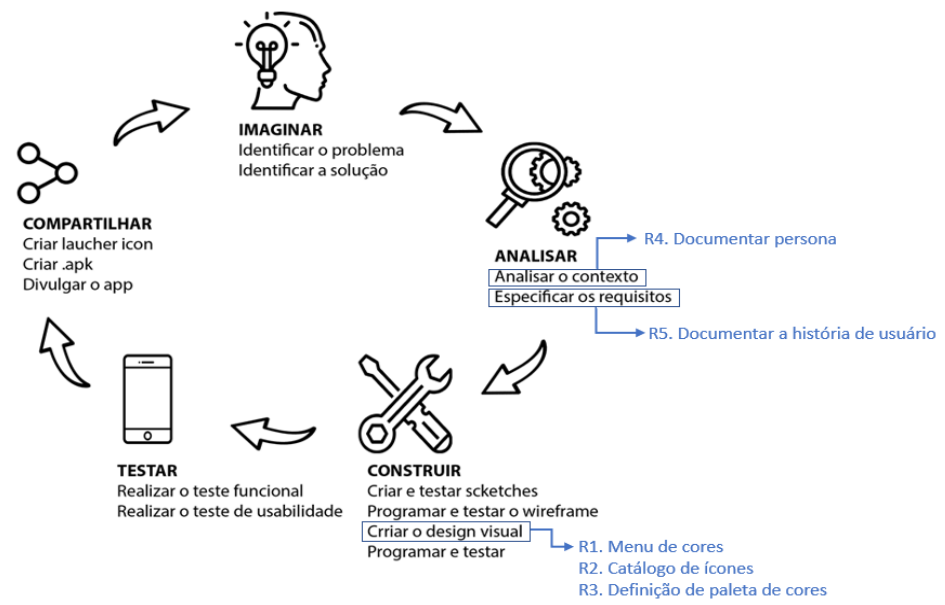

Figura 1. Melhorias identificada no ensino de design de interface.

\subsection{Implementação}

A partir de um fork da versão nb171 do App Inventor do MIT (código aberto) foram implementadas as novas funcionalidades, relacionadas a seguir.

Menu de cores. Foram substituídas as cores do menu pelas cores principais (com o valor de saturação 500) em alinhamento com o Material Design (Figura 2a), permitindo que o usuário possa escolher alguma tonalidade mais clara ou escura da cor selecionada.

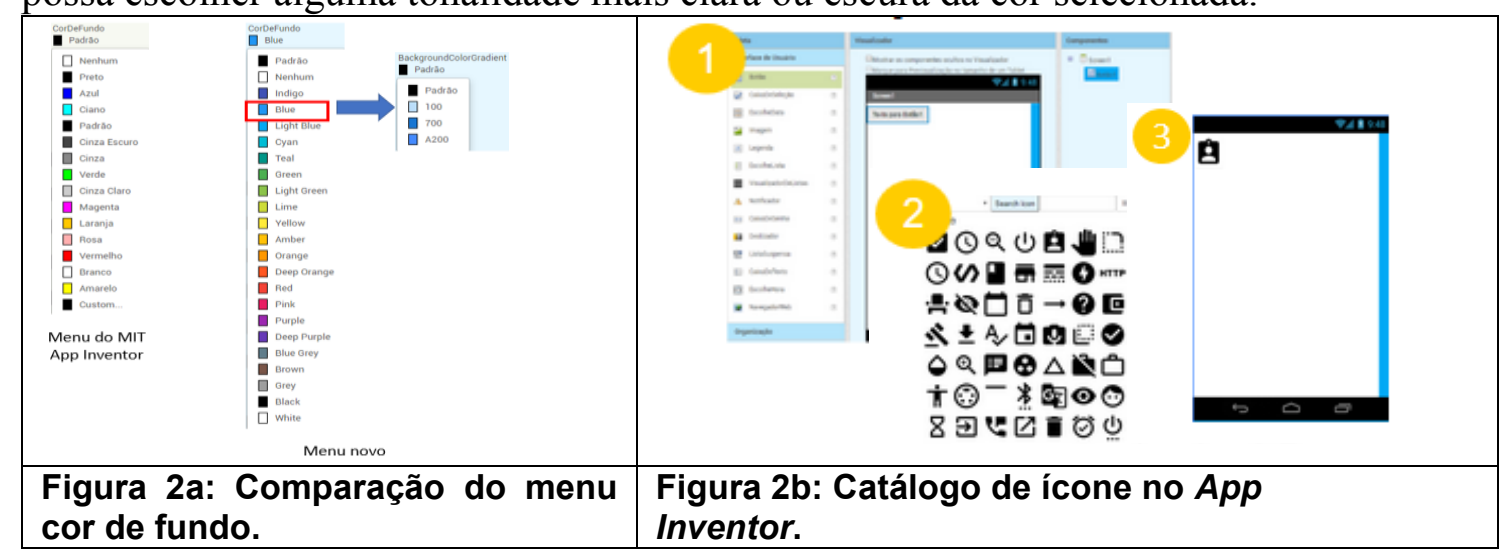

Catálogo de ícones. Foi implementada uma funcionalidade nova que permite inserir diretamente um ícone do catálogo de ícones do Material Design (Figura 2b).

Paleta de cores. Foi implementado um suporte semiautomático à escolha de paleta de cores em 5 etapas (Figura 3a). Definida a paleta de cores, são atualizadas automaticamente as cores de todos os elementos visuais no app sendo desenvolvido.

Documentação de persona e história de usuário. Foi implementada uma funcionalidade nova que permite criar, editar ou excluir personas e histórias de usuário suportando o processo de desenvolvimento de apps (Figura 3b). 
VIII Congresso Brasileiro de Informática na Educação (CBIE 2019)

Anais do XXV Workshop de Informática na Escola (WIE 2019)

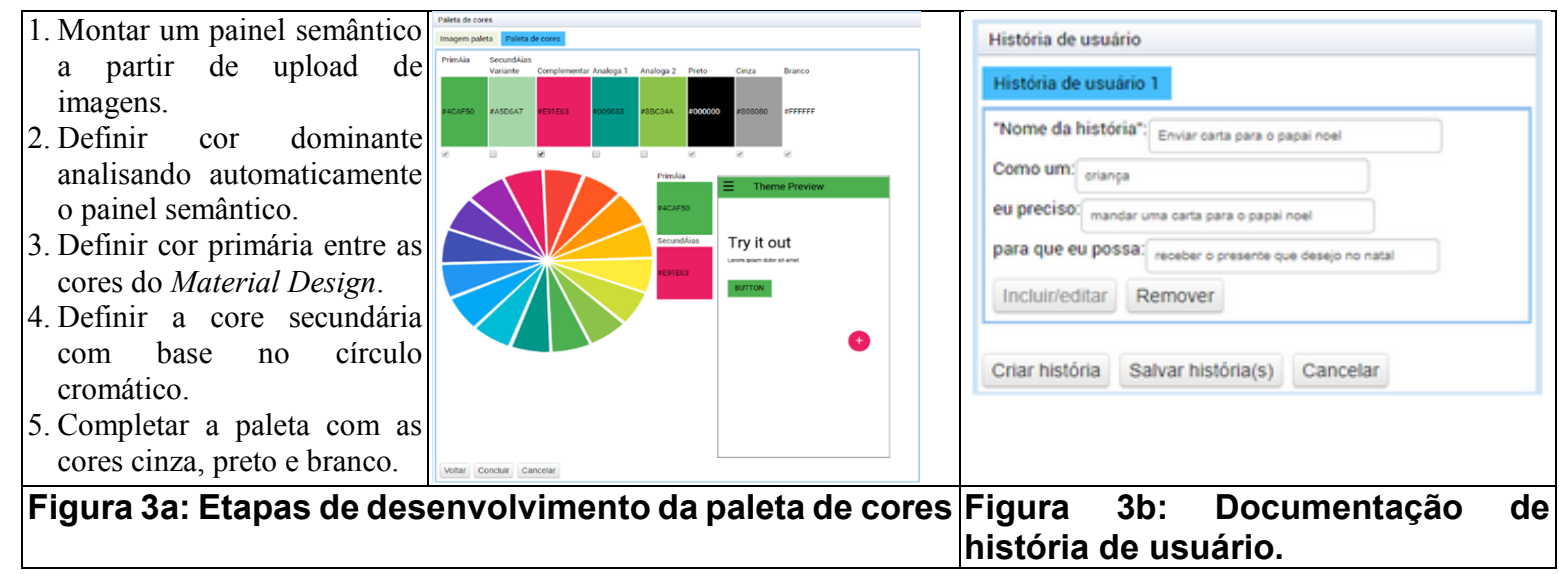

\section{Estudo de caso: aplicação e resultados}

Com o objetivo de obter um feedback inicial das melhorias realizadas no App Inventor foi realizado um estudo de caso. A ferramenta evoluída foi adotada na aplicação da unidade instrucional "Faça um app". Essa unidade instrucional de 8 h/a foi aplicada com 28 alunos da $7^{a}$ fase do ensino médio em 2019 e aborda o ensino de conceitos e práticas básicas de programação como também o design visual de apps. Os alunos programam uma parte de um aplicativo para a visualização de pontos de coleta de tampas PET no âmbito da iniciativa ECOPET na Grande Florianópolis. Em seguida, levando em consideração o pouco tempo disponível pela aplicação nessa disciplina, foi entregue uma versão completa do app já préprogramada no nível wireframe (Figura 4a).

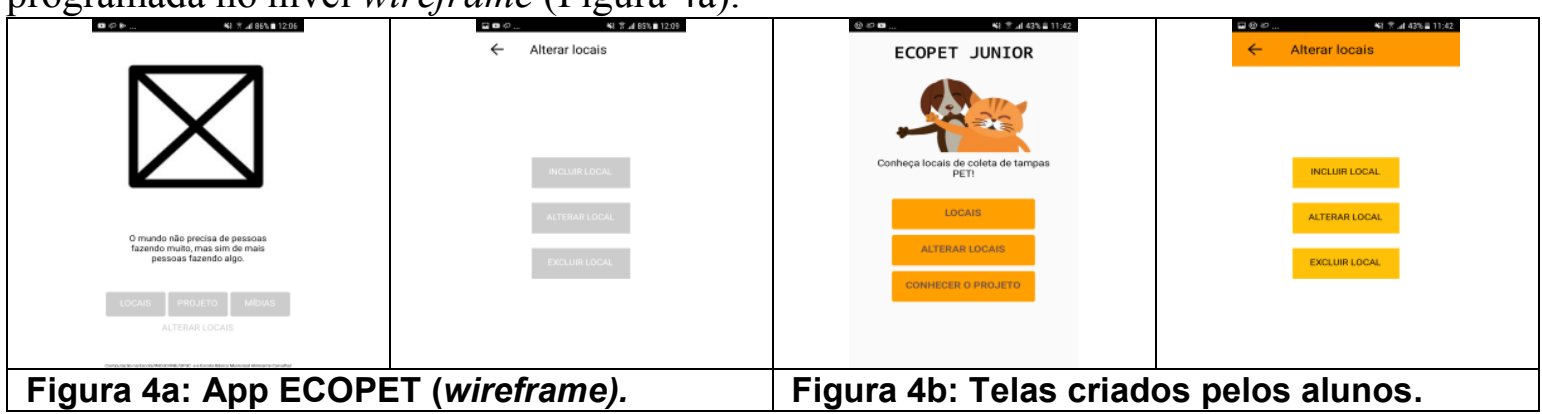

Então, os alunos aprenderam conceitos básicos de design visual e aplicaram principalmente melhorias no App Inventor referente a cores. De forma geral, os aplicativos desenvolvidos pelos alunos apresentaram cores coerentes com seus temas e contraste (Figura 4b). Comparando esses resultados também com apps aleatoriamente escolhidos da Galeria do App Inventor se destacou a maior qualidade do design visual.

Ameaças à validade. Este estudo representa somente uma avaliação preliminar para obter um feedback inicial. Por ser um curso curto, não são abordadas todas as etapas de desenvolvimento, como a análise de requisitos e do contexto. Dessa forma, nessa aplicação não foi possível avaliar as melhorias relacionadas à documentação de histórias de usuário e personas. Como trabalho futuro estamos planejando a realização de um estudo cientificamente mais rigoroso e com uma amostra maior.

\section{Conclusão}

Este artigo apresenta melhorias no App Inventor visando suportar o ensino de design de interface de usuário como parte do ensino de computação na Educação Básica. Essas 
VIII Congresso Brasileiro de Informática na Educação (CBIE 2019)

Anais do XXV Workshop de Informática na Escola (WIE 2019)

melhorias visam tanto o suporte da documentação de artefatos criados no processo de desenvolvimento de apps quanto o design visual em conformidade com diretrizes. Resultados de uma aplicação preliminar, fornecem uma primeira indicação de que as melhorias no App Inventor podem ser úteis e usáveis. Com base nesses resultados positivos, pretendemos continuar a aplicação dessa versão melhorada do App Inventor em futuros cursos.

\section{Agradecimentos}

Este trabalho foi apoiado pelo CNPq (Conselho Nacional de Desenvolvimento Científico e Tecnológico - www.cnpq.br), entidade do governo brasileiro voltada para o desenvolvimento científico e tecnológico.

\section{Referências}

Ahn, S. (2014) "A study on information science curriculum of productivity tools to increase ability for problem solving in elementary and middle school". Korea Association of Information Education, 18(2), p. 235-242.

AIGA. (2013) “The Professional Association for Design”. https://www.aiga.org/.

Basili, V. R e Larman, C. (2003) "Iterative and incremental developments. a brief history", Computer, 36(6), p. 47-56.

CSTA. (2017) “CSTA K-12 Computer Science Standards”, ACM, New York, USA.

Ferreira, M. N. F., et al. (2019). "Learning user interface design and the development of mobile applications in middle school". ACM Interactions, 26(4), p. 66-69.

Garrett, J. J. (2011) "Elements of user experience, the: user-centered design for the web and beyond”. Berkley: New Riders Press.

Google. (2019) "Material Design". https://material.io/guidelines/materialdesign/introduction.html, Junho.

Grover, S. e Pea, R. (2013) "Computational Thinking in K-12: A review of the state of the field". Educational Researcher, 42(1), p. 38-43.

ISO/IEC 9241-210:2011. (2011) "Ergonomia da Interação Humano-sistema: Parte 210: Projeto centrado no ser humano para sistemas interativos". Rio de Janeiro, Brasil.

Pinheiro F. d. P. (2019) "Modelo instrucional para o ensino de Engenharia de Software e Usabilidade voltado ao Ensino Fundamental”. Dissertação, PPGCC, UFSC, Brasil.

Pinheiro, F. d. C. et al. (2018) "Teaching Software Engineering in K-12 Education: A Systematic Mapping Study.” Informatics in Education, 17(2), p.167-206.

Schlatter, T. e Levinson, D. (2013) "Visual usability: Principles and practices for designing digital applications". San Francisco: Morgan Kaufmann.

West-Knights, I. (2017) "Why are schools in China looking west for lessons in creativity". Financial Times.

Yin, R. K. (2009) “Case Study Research: Design and Methods”. SAGE, 4 ed., p. 219. 